\begin{tabular}{|c|c|c|}
\hline \multirow{3}{*}{$\begin{array}{r}\text { Case Reports in } \\
\text { Gastroenterology }\end{array}$} & \multirow{2}{*}{\multicolumn{2}{|c|}{ Case Rep Gastroenterol 2013;7:266-271 }} \\
\hline & & \\
\hline & $\begin{array}{l}\text { DOI: } 10.1159 / 000353635 \\
\text { Published onlIne: June } 22,2013\end{array}$ & $\begin{array}{l}\text { ○ } 2013 \text { S. Karger AG, Basel } \\
\text { 1662-0631/13/0072-0266 } \$ 38.00 / 0 \\
\text { www.karger.com/crg }\end{array}$ \\
\hline & $\begin{array}{l}\text { This is an Open Access article licen } \\
\text { NonCommercial-NoDerivs } 3.0 \mathrm{Li} \\
\text { online version of the article only. }\end{array}$ & $\begin{array}{l}\text { Is of the Creative Commons Attribution } \\
\text { er.com/OA-license), applicable to th } \\
\text { on-commercial purposes only. }\end{array}$ \\
\hline
\end{tabular}

\title{
T1 Colorectal Cancer with Synchronous Liver Metastasis
}

\author{
Kiichi Sugimoto ${ }^{a}$ Masaya Kawai ${ }^{a} \quad$ Kazuhiro Takehara ${ }^{a}$ \\ Yoshihiko Tashiro $^{a}$ Shinya Munakata ${ }^{a}$ Shun Ishiyama ${ }^{a}$ \\ Hiromitsu Komiyama ${ }^{a}$ Makoto Takahashi ${ }^{a}$ Yutaka Kojima $^{a}$ \\ Michitoshi Goto $^{a}$ Yuichi Tomiki $^{a} \quad$ Kazuhiro Sakamoto $^{a}$ Seiji Kawasaki ${ }^{b}$ \\ Departments of ${ }^{a}$ Coloproctological Surgery and ${ }^{b}$ Hepato-Biliary-Pancreatic Surgery, \\ Juntendo University Faculty of Medicine, Tokyo, Japan
}

\section{Key Words}

T1 colorectal cancer · Lymph node metastasis · Synchronous liver metastasis · Distant metastasis · Risk factor

\begin{abstract}
The patient was a 68-year-old man who was admitted to our hospital with a liver tumor. Abdominal ultrasonography and computed tomography revealed a liver tumor $30 \mathrm{~mm}$ in diameter. On colonoscopy, a pedunculated tumor with a central depression $(20 \mathrm{~mm}$ in diameter) was observed in the ascending colon, and this tumor was considered to be invading deeply into the submucosal layer. Right hemicolectomy with D3 lymphadenectomy and partial hepatectomy were performed simultaneously. On histopathological examination of the resected specimen, the tumor was a well-differentiated tubular adenocarcinoma with $3,000 \mu \mathrm{m}$ invasion of the submucosal layer. The liver tumor showed histological findings similar to those of the primary colorectal carcinoma. The pathological stage according to the 7th edition of the TNM classification was stage IV (T1NOM1). Nine months after the operation, computed tomography revealed hepatic hilar lymph node metastases and a great deal of ascites. The patient ultimately died 14 months after the operation.
\end{abstract}

(C) 2013 S. Karger AG, Basel

\section{Introduction}

Colorectal cancer is one of the most common cancers in developed countries [1]. In Japan, it accounts for the largest number of deaths from malignant neoplasms in women and the third largest number in men [1]. However, since the recurrence rate of T1 colorectal

Kiichi Sugimoto

Department of Coloproctological Surgery

Juntendo University Faculty of Medicine

2-1-1 Hongo, Bunkyo-ku, Tokyo 113-8421 (Japan)

E-Mail ksugimo@juntendo.co.jp 
Sugimoto et al.: T1 Colorectal Cancer with Synchronous Liver Metastasis

cancer (invasion no deeper than the submucosa) without lymph node metastasis is approximately $1 \%$, the outcome of $\mathrm{T} 1$ colorectal cancer is considered to be good [1]. On the other hand, the incidence of synchronous liver metastases in colorectal cancer has been reported to be about 10\% [2], yet T1 colorectal cancer with synchronous liver metastasis is considered to be rare $[3,4]$. The form of metastases from T1 colorectal cancer is mainly lymph node metastases. A few patients exhibit hematogenous metastases, such as liver metastases. Therefore, there have been many reports of lymph node metastases, but few reports of the risk factors of synchronous or metachronous distant metastases from T1 colorectal cancer. We report a case of $\mathrm{T} 1$ colorectal cancer of the ascending colon with synchronous liver metastasis.

\section{Case Report}

The patient was a 68-year-old Japanese man who was admitted to our hospital with a liver tumor that had been detected by abdominal ultrasonography. His medical history included hypertension, diabetes mellitus and gout. Physical examination indicated no significant findings. Laboratory tests showed: erythrocyte count $484 \times 10^{4} / \mathrm{mm}^{3}$ (normal $420-$ 554), hemoglobin $15.9 \mathrm{~g} / \mathrm{dl}$ (normal 13.8-16.6), leukocyte count 8,600/ $\mathrm{mm}^{3}$ (normal 3,5009,000 ), platelet count $21.8 \times 10^{4} / \mathrm{mm}^{3}$ (normal 15.5-36.5), serum total protein $6.5 \mathrm{~g} / \mathrm{dl}$ (normal 6.3-8.1), total bilirubin $0.69 \mathrm{mg} / \mathrm{dl}$ (normal 0.3-1.2), aspartate aminotransferase $28 \mathrm{IU} / \mathrm{l}$ (normal 9-38), alanine aminotransferase 27 IU/l (normal 4-36), alkaline phosphatase $224 \mathrm{IU} / \mathrm{l}$ (normal 115-359), $\gamma$-glutamyl transpeptidase $53 \mathrm{IU} / \mathrm{l}$ (normal 4-68), blood urea nitrogen $16 \mathrm{mg} / \mathrm{dl}$ (normal 9-21), and creatinine $0.92 \mathrm{mg} / \mathrm{dl}$ (normal 0.60-1.20). Hepatitis B surface and hepatitis $\mathrm{C}$ antibody were negative. Carcinoembryonic antigen and CA19-9 were both elevated at $23.9 \mathrm{ng} / \mathrm{ml}$ (normal 0-3) and $51 \mathrm{U} / \mathrm{ml}$ (normal 0-37), respectively.

Abdominal ultrasonography revealed a hyperechoic mass in segment 7 of the liver, $30 \mathrm{~mm}$ in diameter, with a circumferential hypoechoic area. Abdominal computed tomography also showed mild enhancement of a liver tumor $30 \mathrm{~mm}$ in diameter at the portal phase (fig. 1a). On colonoscopy, a pedunculated tumor with a central depression $(20 \mathrm{~mm}$ in diameter) was observed in the ascending colon, and this tumor was considered to be invading deeply into the submucosal layer (fig. 1b). Abdominal magnetic resonance imaging showed the liver tumor as a low-intensity mass in the T1 emphasis phase (fig. 1c), as a high-intensity mass in the T2 emphasis phase (fig. 1d) and as a strongly high-intensity mass by administering the contrast medium ferumoxide (fig. 1e). T1 colorectal carcinoma with liver metastasis was suspected, and right hemicolectomy with D3 lymphadenectomy and partial hepatectomy were performed simultaneously (fig. 2a, b). On histopathological examination of the resected specimen, the tumor was a well-differentiated tubular adenocarcinoma with $3,000 \mu \mathrm{m}$ invasion of the submucosal layer (fig. 2c, d) and venous permeation without tumor budding or lymph node metastasis. The liver tumor showed histological findings similar to those of the primary colorectal carcinoma (fig. 2e). The pathological stage according to the 7th edition of the TNM classification was stage IV (T1N0M1). The patient had an uneventful postoperative course. He took 5-FU drugs orally for 6 months as a postoperative adjuvant chemotherapy. Nine months after the operation, elevation of serum carcinoembryonic antigen was recognized. Computed tomography revealed hepatic hilar lymph node metastases and a great deal of ascites. His general condition worsened, and the patient ultimately died 14 months after the operation. 
Sugimoto et al.: T1 Colorectal Cancer with Synchronous Liver Metastasis

\section{Discussion}

A multicenter study in Japan reported that 153 (8.5\%) of 1,806 patients with T1 colorectal cancer had lymph node metastases and $40(2.2 \%)$ of 1,806 patients had synchronous or metachronous distant metastases [3]. Among them, the incidence of liver metastases was $1.2 \%$. Furthermore, when the focus was restricted to synchronous liver metastases, the incidence was $0.2 \%$. Okano et al. [4] also reported that the incidence of liver metastases from $\mathrm{T} 1$ colorectal cancer was $3.3 \%$ and that of synchronous liver metastases $0.9 \%$. Thus, the form of metastases from T1 colorectal cancer is mainly lymph node metastases. However, a few patients exhibit hematogenous metastasis, such as liver metastases. Moreover, synchronous liver metastases are thought to be extremely rare. Therefore, to date, there have been many reports of lymph node metastases but few reports of distant metastases.

With respect to the risk factors of lymph node metastasis in T1 colorectal cancer, Sakuragi et al. [5] reported that the depth of submucosal invasion $(\geq 2,000 \mu \mathrm{m})$ and lymphatic invasion significantly predicted the risk of lymph node metastasis in multivariate analysis. In addition, the depth of submucosal invasion $(\geq 1,000 \mu \mathrm{m})$ [6], lymphovascular invasion [6], histological grade $[7,8]$ and budding at the invasive front of the tumor $[7,8]$ were thought to be significantly associated with lymph node metastasis. On the other hand, with respect to the risk factors of synchronous or metachronous distant metastases from T1 colorectal cancer, nodal metastases, depth of invasion and venous invasion are considered to be risk factors [3]. Okano et al. [4] also reported that venous invasion was closely related to liver metastasis. These risk factors of distant metastasis are often common to those of lymph node metastases. It is considered that the highly malignant potential of T1 colorectal cancer, which can invade the submucosal layer broadly or exhibit poor differentiation, allows the tumor to invade the venous plexus of the submucosal layer and then develop distant metastases. In our case, submucosal invasion $(3,000 \mu \mathrm{m})$ and venous invasion were recognized as risk factors. Consequently, this case had multiple risk factors of distant metastasis.

Liver resection for resectable liver metastases is recommended as the most effective therapy [9]. It has also been reported that the 5-year survival rate after liver resection in patients with synchronous liver metastases is $19-38 \%$, and that the prognosis of patients with resectable synchronous liver metastases is better than that of those with non-resectable liver metastases [10]. However, even after curative resection, some cases have a poor prognosis for recurrence in the remnant liver and exhibit extrahepatic recurrence. In our case, postoperative adjuvant chemotherapy was undertaken for 6 months, however the prognosis was poor because of extrahepatic recurrence 9 months after the operation. Hayashi et al. [11] reported that extrahepatic recurrence was a prognostic factor after curative liver resection and noted that postoperative adjuvant systemic chemotherapy is necessary for patients with synchronous liver metastases because liver metastases should be considered equivalent to systemic disease. Moreover, expert consensus statements by the American Hepato-Pancreato-Biliary Association also concluded that adjuvant systemic chemotherapy after curative liver resection must be supported because most patients with synchronous liver metastases concurrently exhibit extrahepatic metastases [12]. However, the regimens and periods of postoperative adjuvant systemic chemotherapy were not fixed because of the absence of consensus for adjuvant chemotherapy after curative liver resection. Further investigations are necessary in order to judge the usefulness of postoperative adjuvant chemotherapy. There are few well-organized reports because T1 colorectal cancer with synchronous liver metastasis is extremely rare. On the other hand, the prognosis is often poor in patients with metachronous liver metastases after surgery for T1 colorectal cancer [13-15]. Therefore, it is predictable that the prognosis of patients with synchronous 
Sugimoto et al.: T1 Colorectal Cancer with Synchronous Liver Metastasis

liver metastasis is also poor. It is necessary to establish postoperative surveillance and a plan for adjuvant chemotherapy for T1 colorectal cancer with synchronous liver metastasis.

We consider this case to be rare, and it is necessary to accumulate more cases to further elucidate the risk factors for liver metastasis from T1 colorectal cancer.

\section{Disclosure Statement}

The authors declare no conflict of interests for this article.

\section{References}

1 Watanabe T, Itabashi M, Shimada Y, Tanaka S, Ito Y, Ajioka Y, et al: Japanese Society for Cancer of the Colon and Rectum (JSCCR) guidelines 2010 for the treatment of colorectal cancer. Int J Clin Oncol 2012;17:1-29.

-2 Nordlinger B, Parc R, Delva E, Quilichini MA, Hannoun L, Huguet C: Hepatic resection for colorectal liver metastases. Influence on survival of preoperative factors and surgery for recurrences in 80 patients. Ann Surg 1987;205:256-263.

3 Kodaira S, Yao T, Nakamura K, Watanabe E, Maruyama M, Ushio K, et al: Multi-institutional survey of submucosal colorectal cancer with metastases (in Japanese with English abstract). Stomach Intestine 1994;29:1137-1142.

4 Okano K, Shimoda T, Matsumura Y: Clinicopathological and immunohistochemical study of early colorectal cancer with liver metastases. J Gastroenterol 1999;34:334-340.

5 Sakuragi M, Togashi K, Konishi F, Koinuma K, Kawamura Y, Okada M, et al: Predictive factors for lymph node metastasis in T1 stage colorectal carcinomas. Dis Colon Rectum 2003;46:1626-1632.

6 Yamamoto S, Watanabe M, Hasegawa H, Baba H, Yoshinare K, Shiraishi J, et al: The risk of lymph node metastasis in T1 colorectal carcinoma. Hepatogastroenterology 2004;51:998-1000.

7 Wang HS, Liang WY, Lin TC, Chen WS, Jiang JK, Yang SH, et al: Curative resection of T1 colorectal carcinoma: risk of lymph node metastasis and long-term prognosis. Dis Colon Rectum 2005;48:1182-1192.

8 Yamauchi H, Togashi K, Kawamura YJ, Horie H, Sasaki J, Tsujinaka S, et al: Pathological predictors for lymph node metastasis in T1 colorectal cancer. Surg Today 2008;38:905-910.

-9 Choti MA, Sitzmann JV, Tiburi MF, Sumetchotimetha W, Rangsin R, Schulick RD, et al: Trends in long-term survival following liver resection for hepatic colorectal metastases. Ann Surg 2002;235:759-766.

10 de Santibanes E, Lassalle FB, McCormack L, Pekolj J, Quintana GO, Vaccaro C, Benati M: Simultaneous colorectal and hepatic resections for colorectal cancer: postoperative and longterm outcomes. J Am Coll Surg 2002;195:196-202.

11 Hayashi M, Inoue Y, Komeda K, Shimizu T, Asakuma M, Hirokawa F, et al: Clinicopathological analysis of recurrence patterns and prognostic factors for survival after hepatectomy for colorectal liver metastasis. BMC Surg 2010;10:27.

12 Bartlett DL, Berlin J, Lauwers GY, Messersmith WA, Petrelli NJ, Venook AP: Chemotherapy and regional therapy of hepatic colorectal metastases: expert consensus statement. Ann Surg Oncol 2006;13:1284-1292.

13 Bamba Y, Itabashi M, Hirosawa T, Ogawa S, Seshimo A, Shirotani N, et al: Follow-up and recurrence of T1 colorectal cancer. Int Surg 2006;91:12-16.

14 Kobayashi H, Mochizuki H, Morita T, Kotake K, Teramoto T, Kameoka S, et al: Characteristics of recurrence after curative resection for T1 colorectal cancer: Japanese multicenter study. J Gastroenterol 2011;46: 203-211.

15 Iida S, Hasegawa H, Okabayashi K, Moritani K, Mukai M, Kitagawa Y: Risk factors for postoperative recurrence in patients with pathologically T1 colorectal cancer. World J Surg 2012;36:424-430. 
Sugimoto et al.: $\mathrm{T1}$ Colorectal Cancer with Synchronous Liver Metastasis

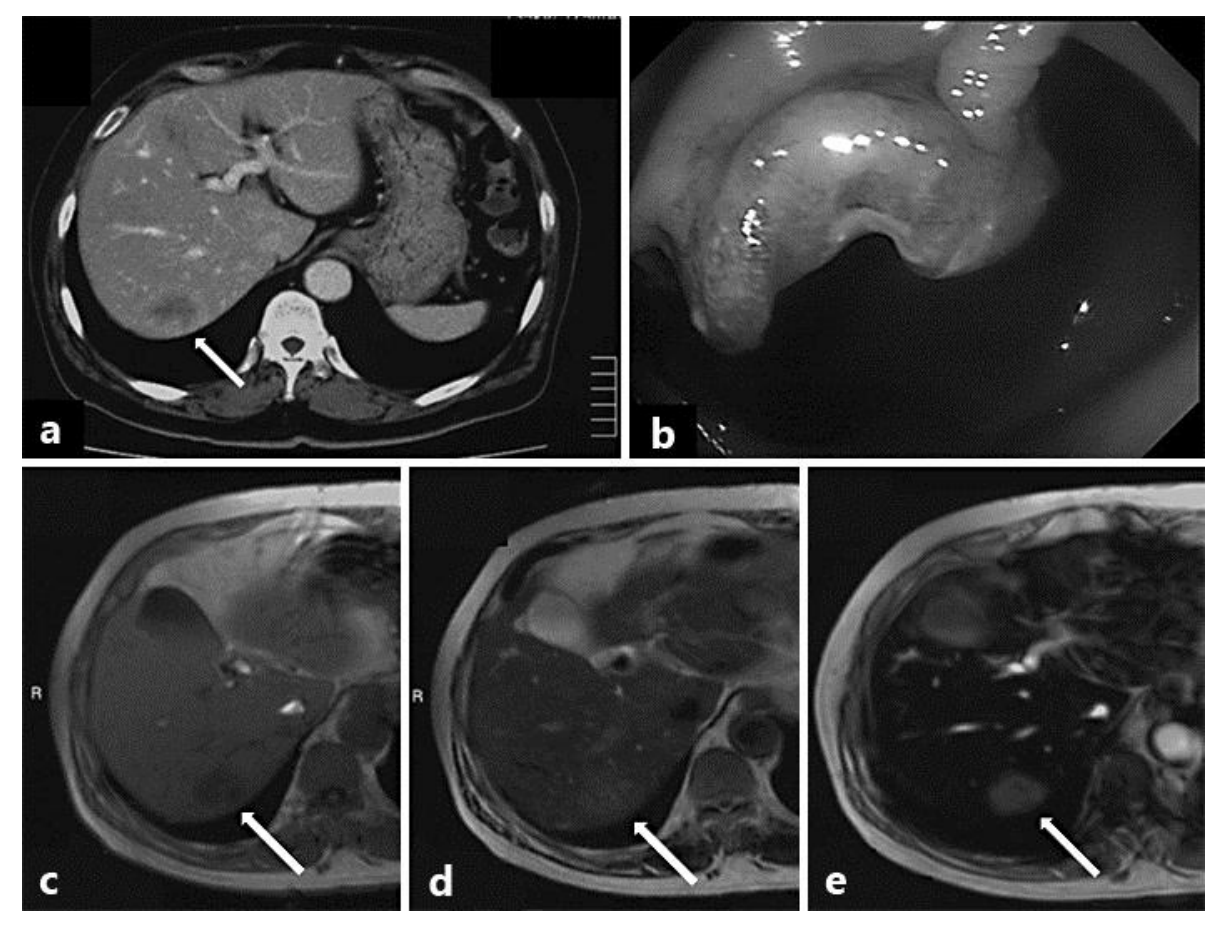

Fig. 1. a Abdominal computed tomography showed mild enhancement of a liver tumor $30 \mathrm{~mm}$ in diameter. b On colonoscopy, a pedunculated tumor with a central depression (20 $\mathrm{mm}$ in diameter) was observed in the ascending colon. c-e Abdominal magnetic resonance imaging showed the liver tumor as a low-intensity mass in the T1 emphasis phase (c), as a high-intensity mass in the T2 emphasis phase (d) and as a strongly high-intensity mass by administering the contrast medium ferumoxide (e). 

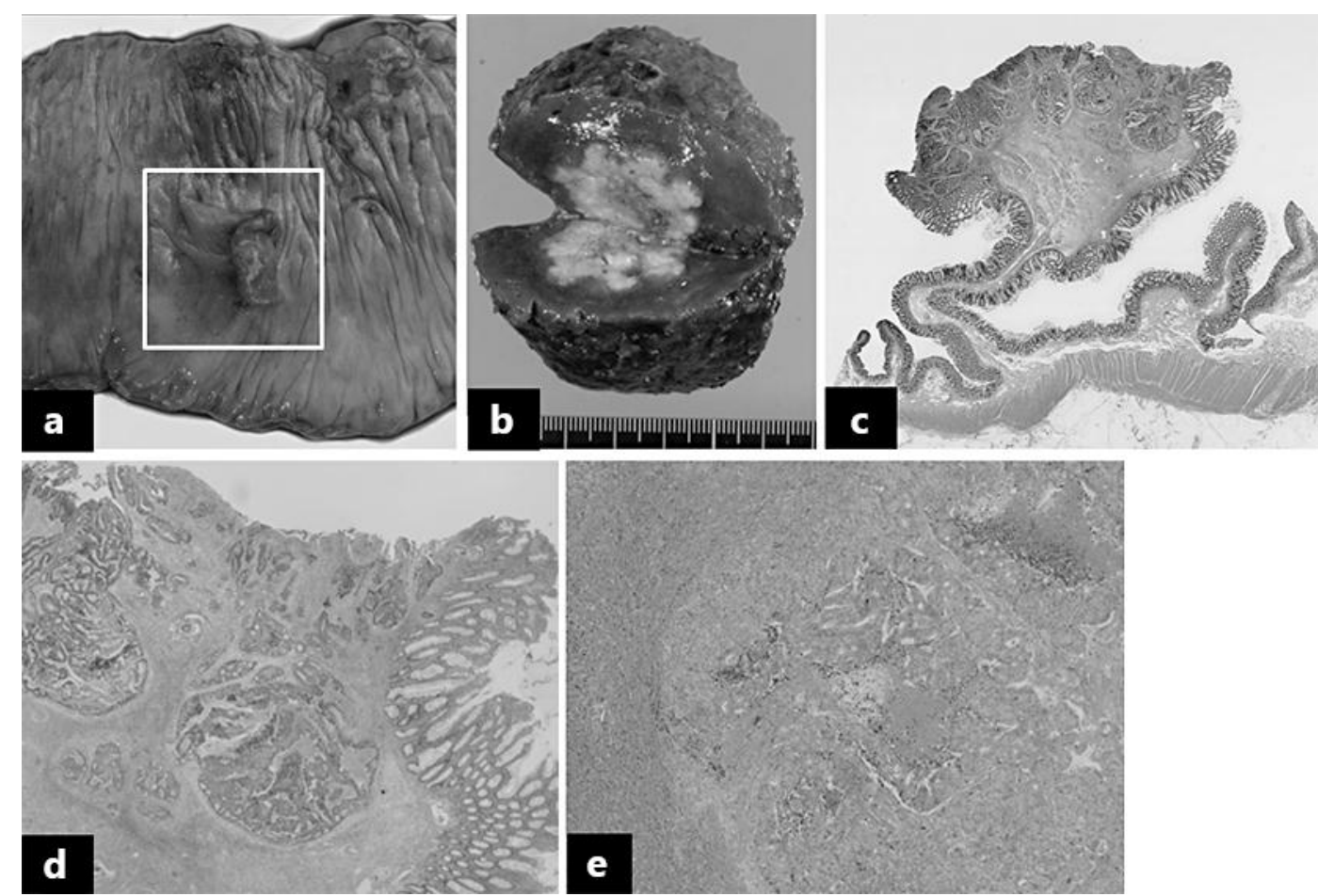

Fig. 2. a, b Right hemicolectomy and partial hepatectomy were performed simultaneously. c, $\mathbf{d}$ The tumor was a well-differentiated tubular adenocarcinoma with $3,000 \mu \mathrm{m}$ invasion of the submucosal layer (c: loupe; d: HE, $\times 40$ ), without tumor budding or lymph node metastasis. e The liver tumor showed histological findings similar to those of the primary colon carcinoma $(\mathrm{HE}, \times 40)$. 